\title{
Automation in Plant Factory with Labor-saving Conveyance System
}

\author{
Minoru TOKIMASA ${ }^{1}$ and Yoshifumi NisHIURA ${ }^{2}$ \\ ${ }^{1}$ Itoh Denki Co.,Ltd., 1146-2 Asazuma-cho, Kasai, Hyogo 679-0180, Japan \\ 2 Osaka Prefecture University, 1-1 Gakuen, Sakai, Osaka, 599-8531, Japan
}

(Received April 19, 2014; Accepted March 31, 2015)

\begin{abstract}
Within current plant factories, it is inevitable for people to enter the cultivation rooms. Along with hygiene issues such as prevention from bacteria and contamination, safety consideration for working on the upper level of the shelves are required. Also the labor cost management directly connected with running costs is also an important yet difficult problem to solve. As one way to solve these problem areas such as, hygiene, employee safety, labor cost management, the automatic culture bed transportation system was evaluated. The automated transportation system designed for the contact with the cultivation environment integrates monitoring management with warehouse control system, remote system control, energy saving and cost reduction techniques with shuttle transportation robots driven by brushless motors $(24 \mathrm{~V})$. This fabricated system with respect to hygiene, employee safety, and labor cost management was found advantageous compared to conventional plant factories. If employees without automatic machines harvest 5000 plants per day, labor running costs will require 25 to 33 employees working one eight hour day, with a total working time of 200 to $264 \mathrm{~h}$. Furthermore, 63-83 hours out of the total (200-264 hours) is transportation work (31\%). The transportation work of 7.8 to 10.4 people out of the 25-33 working one eight h day on 5000 plants per day was witnessed to be automated.
\end{abstract}

Keywords : automation, conveyance system, culture bed transportation, labor cost management, warehouse control system

\section{INTRODUCTION}

Plant factory basics and facts were reported by Takatsuji (1996). The majority of current cultivation methods in plant factories are floating system where the cultivation panels float on water in a container. The panels are removed from the harvesting side by hand, while the new panels are pushed on by hand from the planting side. At the moment, the work is basically done by workers inside the cultivation room. Therefore, it is necessary to solve hygiene issues such as bacteria prevention, and safety considerations for work on the upper shelves. Also, it is important to solve the problem as to how to reduce labor running costs (Now, 5 workers / 1,000 plants, 5,000 yen / day). Low cost artificial type plant factories with total system control were discussed by Takayanagi (2000).

As one way to solve these problem areas such as hygiene, employee safety, and labor cost management, the automatic culture bed transportation system was fabricated and examined. The automation of plant factory operations was discussed by Ogura (2011).

The automated cultivation transport system reported in this paper utilizes the transportation technology as a labor saving structure, intended to innovative and revolutionary closed plant factory systems.

To achieve labor saving employee reduction and safety for nutrient film technique (NFT) multistage cultivation, the system requires automated culture bed loading from the planting side, automated unloading from the har- vesting side, and automated transportation for moving forward to erase the unused spaces. When the system is in use, the only employees work is to set the culture beds on the warehouse entrance plate for planting.

Thus, this system was designed to keep employees safe from working in high-places and to reduce the labor cost of transportation from the planting entrance to the harvest room. Also, hygiene management can be improved and maintained because employee entrance into the cultivation room is limited. The adopted conveyor equipment in this system has the latest network and control system that can communicate the cultivation shelves operation conditions in real time.

Operation instructions and status confirmations such as continuous automated delivery, and trouble detection can be remotely controlled from a centralized location, because the position of the cultivation shelves can be managed. These methods combined are considered to offer labor savings benefits.

From the above items, significant running cost reductions can be achieved, and operating a mass production plant factory becomes feasible.

\section{TRANSPORT SYSTEM CONFIGURATION}

Figure1 shows the system's configuration model. This transportation system mainly consists of shuttle transportation robots and two elevators, one set on the planting side and one on the harvest side. The shuttle transportation robot freely travels horizontally along the cultivation shelf.

Corresponding author: Yoshifumi Nishiura, fax +81-72-254-9428, e-mail : nishiura@bioinfo.osakafu-u.ac.jp 


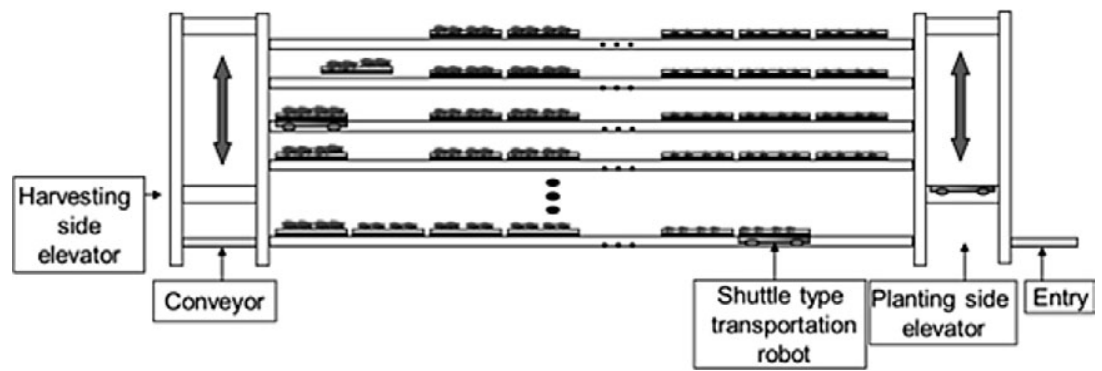

Fig. 1 Basic structure of the transportation system. Right part is planting side elevator. Middle part is the cultivation shelf where the transfer shuttle type transportation robots carry culture beds. Left part is harvest side elevator connected to conveyor.

The elevators carry the shuttle transportation robots from planting and harvesting ground levels to the cultivation shelves. The planted cultivation bed is put on shuttle transportation robot at the ground level planting side by hand, then the shuttle transportation robot with the bed is moved into the elevator. The elevator is moved to the entrance of cultivation shelf, then the shuttle transportation robot with the bed is moved onto the cultivation shelf. On the cultivation shelf the plants grow up while moving to the harvest side elevator. At last when the harvest time has come, the cultivation bed is moved into the harvest side elevator with a shuttle transportation robot, and the shuttle transportation robot lowers the bed onto the harvest side elevator and returns to the cultivation shelf, while the bed on the harvest side elevator is carried down to the ground conveyor.

Figure 2 shows the process after entering the cultivation shelf. At the beginning, the planting unit places the cultivation bed onto the shuttle transportation robot at the entrance, then the shuttle transportation robot enters the elevator (Step 1 in Fig. 2), and is lifted up (Step 2 in Fig. 2) to the designated shelf entrance where it stops (Step 3 in Fig. 2). From there, the shuttle transportation robot travels onto the cultivation shelf, transports the cultivation bed to

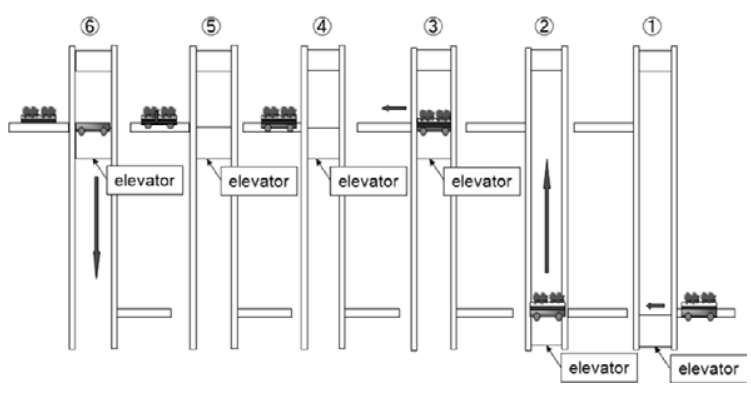

Fig. 2 Process after entering the cultivate shelf. the predetermined location (Step 4 in Fig. 2), and places it on the shelf (Step 5 in Fig. 2). Then, the shuttle transportation robot returns to the elevator and to the entrance (Step 6 in Fig. 2), completing the operation.

Figure 3 shows the process from the cultivation shelf to the harvest conveyor. When it is time to harvest, the shuttle transportation robot moves beneath the target cultivation bed, carries it to the harvest side elevator (Step 1 and 2 in Fig. 3), and unloads the culture bed onto the elevator (Step 3 in Fig. 3). Then, the shuttle transportation robot returns to the cultivation shelf (Step 4 in Fig. 3). The culture bed is taken down to the harvest conveyor (Step 5 in Fig. 3) which is connected to the cutting and packaging room, completing the delivery operation of the culture bed.

\section{Shuttle transportation robot features}

The operation model for the shuttle transportation robot is shown in Fig. 4. The shuttle transportation robot not only moves horizontally as shown in Fig. 1, but also has short-stroke lifting equipment. This added equipment can raise and lower the cultivation bed. In Fig. 4 at first the

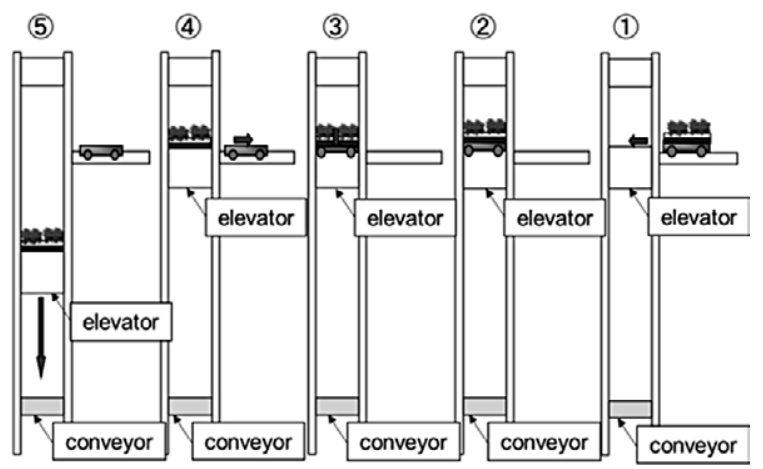

Fig. 3 Process from the cultivate shelf to the harvest conveyor.

\begin{tabular}{|c|c|c|}
\hline 1 moves under the cultivation bed & 2 Stops & $\begin{array}{l}\text { 3. Lifts the cultivation bed } \\
\text { up from the shelf }\end{array}$ \\
\hline
\end{tabular}

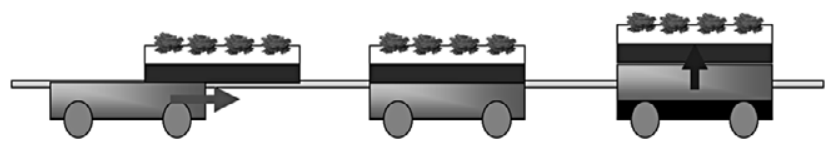

Fig. 4 Shuttle transportation robot motion. 

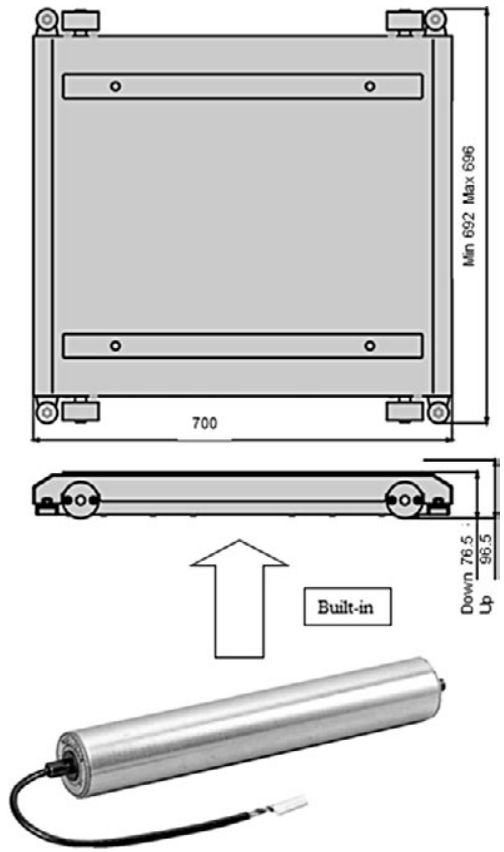

Fig. 5 Shuttle transportation robot structure. Upper side: Horizontal travel and lift arm mechanism. Lower side: Highly efficient, small diameter DC $24 \mathrm{~V}$ driven brushless motor for drive source. shuttle transportation robot is moved under the culture bed and is stopped at the precise lifting point, then the shortstroke lifting equipment above the shuttle transportation robot lifts up the culture bed, and the shuttle transportation robot with the bed can be carried anywhere without interfering with other beds. With one shuttle transportation robot, a cultivation bed can be raised, lowered, or positioned with high efficiency. In addition, the shuttle transportation robot can place the cultivation bed on any cultivation shelf using the elevator, so there is no need to install a shuttle transportation robot for each shelf. The shuttle transportation robot shown in Fig. 5 has two drive sources for vertical lift and horizontal travel. Each drive source uses a highly efficient brushless DC motor $(24 \mathrm{~V})$ at the minimum required power output $(80 \mathrm{~W}$ or less). Because power is obtained from storage batteries, the shuttle transportation robot is cordless. The motors have an output low enough to be stopped by human hand and offer a significant reduction in power consumption, along with prevention of accidents such as electric shock or external contact.

Shuttle transportation robot control system

As explained before, the shuttle transportation robot has a communication function which operates wirelessly with instructions from the warehouse control system (WCS). Figure 6 indicates the shuttle transportation robot

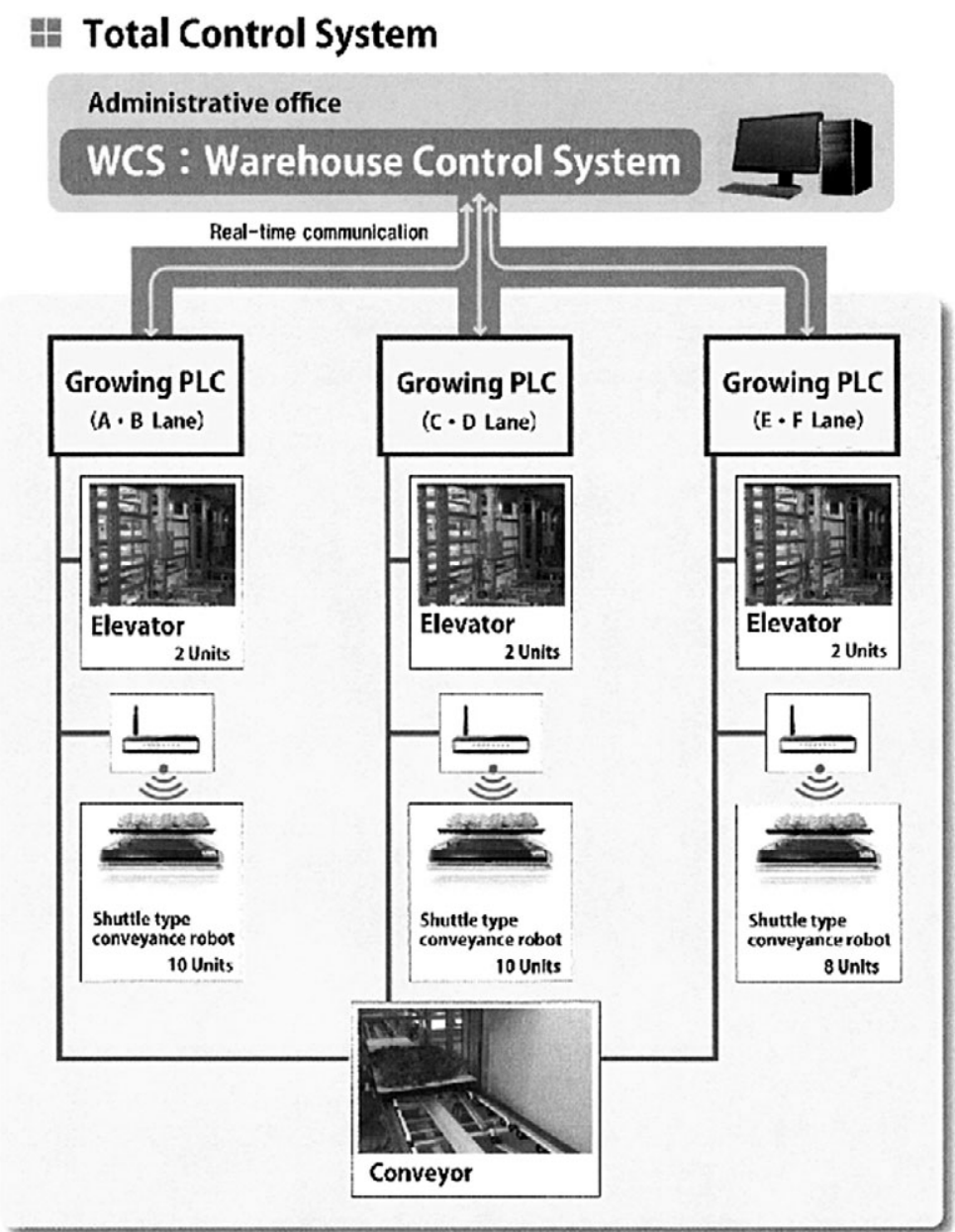

Fig. 6 Shuttle transportation robot control system structure. 
control system construction. This system consists of the elevator controller for loading and/or unloading of the culture beds, the shuttle transportation robot moving controller, and the harvest conveyor controller. Both the elevator controller and the shuttle transportation robot moving controller have three blocks, as there are six culture bed lanes (as shown A, B, C, D, E, F in Fig. 6). As all the culture beds for harvest are gathered in the cutting and package room, one harvest conveyor controller is applied to connect the six lane cultivation shelves. The commands and information can be communicated from both office terminal and factory terminal via WCS server, so locations and situations of the culture beds' loading/unloading can be controlled from anywhere. The bed transfer system within the cultivation shelves can be controlled remotely. With the WCS it is possible to centralize management for continued automatic discharging and for detecting troubles during transport. When conveying the cultivation bed, it is necessary to stop the nutrient supply and drain in the cultivation bed because the NFT culture must always be irrigated. If vibrations or impact shock occur during transportation, the excess nutrient solution spills through the drain outlet, and the cultivation bed on the shuttle transportation robot will slip with sudden start or stop. Control functions were added accordingly to slowly start and stop the shuttle transportation robot in order to inhibit the vibration and impact on the cultivation bed during transportation.

\section{SHUTTLE TRANSPORTATION ROBOT TRANSPORT SIMULATION}

\section{Horizontal and vertical transportation test equipment and features}

The proposed conveyor system is planned to produce 5,000 plants a day at Osaka Prefecture University (Building C22). Figure 7 shows Osaka Prefecture University Building C22 layout. This building is a plant factory that grows plants from seeding through harvesting. This conveyor system is adapted to multistage cultivation shelves without a seeding/nursing room and harvest room.

Therefore, the shuttle transportation robots must be able to transport 5,000 lettuce a day, one nutrient solution group in about $30 \mathrm{~min}$. The cultivation bed used is roughly $1,200 \times 700 \mathrm{~mm}$ in size and about $10 \mathrm{~kg}$ in weight. The number of cultivation days using the cultivation shelves (4 rows $25 \mathrm{~m}$ long, 18 shelves and 2 rows $25 \mathrm{~m}$ long 16

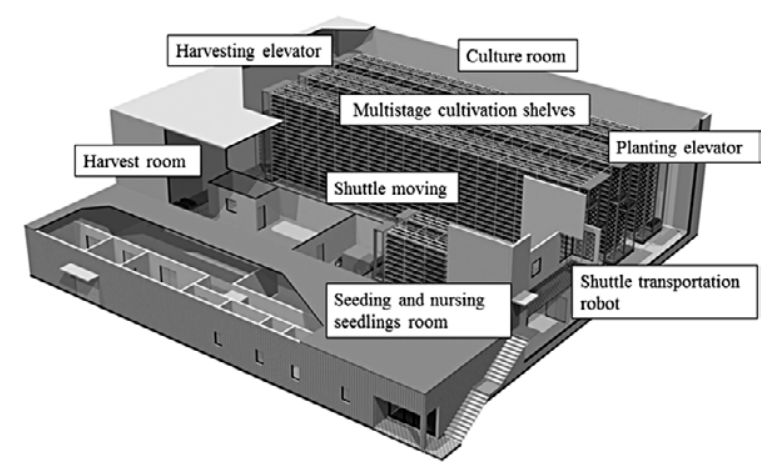

shelves, each 36 cultivation beds per 1 row of 1 shelf) is 18 . The number of cultivation trays necessary to transport 5,000 plants a day is 200 for loading and unloading, while 3,700 are used for moving forward to erase the unused spaces. During transportation, it is necessary to stop the nutrient solution circulation. The liquid feed is stopped, and after draining the transport starts. When the transport is completed, the solution feed supply and drain is resumed. The irrigation stop time is estimated to be about $30 \mathrm{~min}, 10$ min drainage time, $31 \mathrm{~min}$ for entering and dispatching the culture beds from cultivation shelves, 21 min transportation after shipment for moving forward to erase the unused spaces. Without spilling the nutrient solution or causing the cultivation bed to slip, the horizontal speed of the shuttle transportation robot is roughly $2.9 \mathrm{~m} \mathrm{~min}^{-1}$ (max speed $14 \mathrm{~m} \mathrm{~min}^{-1}$ ) while the elevator speed is roughly $20 \mathrm{~m}$ $\min ^{-1}$. With these conditions 28 shuttle robots are used. Through adoption of this system, multistage cultivation can be performed without employees working in high places. In addition, monitoring functions using WCS for sensing trouble can ensure both work safety and reduced labor costs.

Human contact with the cultivation environment can only occur when employees load the transplanted cultivation bed onto the shuttle transportation robot. After exiting, the shuttle transportation robot passes on the conveyor through a shutter to the harvest room. In the harvest room the cultivation bed is removed from the conveyor. Therefore, contact with the cultivation environment is minimized to a short time, and hygiene prevention can also be ensured.

Shuttle transportation robot power consumption

From the viewpoint of reduced labor and energy, the power consumption between a standard roller conveyor and the shuttle conveyor was compared. As stated before, the energy usage of the standard roller conveyor equipment in Building C22 is $29.3 \mathrm{kWh}$ a day (power usage while operational: $0.1 \mathrm{kWh}$, power while in standby: $29.2 \mathrm{kWh}, 9 \mathrm{~h}$ applying current). In the shuttle transportation robot's case, the energy usage is $4.8 \mathrm{kWh}$ a day $(2.9 \mathrm{kWh}$ while charging, and $1.9 \mathrm{kWh}$ from the charging equipment when in standby mode), and is roughly 0.164 times that of a standard roller conveyor. From this data, the benefits of this system were acknowledged, in terms of labor cost reduction and energy saving.

Transportation equipment safety evaluation for contact

The transportation equipment is forced to stop when unexpected contact with anything occurs. The steady state force was measured when contacted by an object. By using a push pull gauge (IMADA Co., Ltd. DS2-200N), the prohibitive power to stop the shuttle transportation robot running at a constant speed of $20 \mathrm{~m} \mathrm{~min}^{-1}$ was measured as impact force. The resulting force was $110 \mathrm{~N}$. It was found that a shuttle transportation robot in motion could be stopped with a $10 \mathrm{~kg}$ force, $1 / 3$ of what is normally applied from an employee.

Fig. 7 Osaka Prefecture University Building C22 layout. 


\section{AUTOMATION IN PLANT FACTORY}

\section{DISCUSSION AND FUTURE CHALLENGES}

In this paper, hygiene, employee safety, and worker cost consumption issues within plant factories were examined with respect to the multistage cultivation transportation system intended to be labor saving, labor reducing, and safe. The results show the automated conveyor and connection with the cultivation environment using the system design, integrated monitoring management from the WCS, remote controlled energy saving, low-cost shuttle transportation robot with $(24 \mathrm{~V})$ brushless motor. From these results, the validity of this system was verified with respect to hygiene prevention, employee safety, and labor cost management compared to the traditional plant factories. If employees without automatic machines harvest 5,000 plants per day, labor running costs will require 25 to 33 employees working one eight hour day (Yamamoto, 2013), with a total working time of 200 to $264 \mathrm{~h}$. Furthermore, $63-83 \mathrm{~h}$ is spent for transportation work (31\%). The transportation work from 7.8 to 10.4 people out of $25^{-33}$ is automated.

This system will be installed at the plant factory model at the Osaka Prefecture University, where 5,000 leaf lettuce will be produced daily. Demonstrating the practical application will be necessary for evaluating safety and reliability, as well as evaluating initial cost and running cost of this facility. With these multiple evaluations, labor savings such as the automation of harvesting, wrapping with film, packing in the box and automatic cultivation bed washing needs to be advanced. This will drastically reduce the hours required for manual work or the heavily tiring process of washing the cultivation bed, and will allow for a new type plant factory that has never been seen.

\section{REFERENCES}

Ogura, T. 2011. Operation that should be automated and should not be automated in plant factory for leaf vegetables. Sci. High Technol. Agric. 23: 37-43.

Takatsuji, M. 1996. Basic and Facts in Plant Factory. Shokabo, Tokyo, p 1-140.

Takayanagi, E. 2000. Low cost artificial type plant factory with total system. January, Society of Science and High Technology in Agriculture, Tokyo, Science and High Technology in Agriculture Symposium Report, 16, p 55-62.

Yamamoto, H. 2013. Current Situation and Problems in Plant Factory. (in Japanese) Agriculture and Forestry Statistics Publishing Inc., Tokyo, pp 194. 